\title{
The TOTEM experiment at LHC for proton-proton cross section measurements
}

\author{
Francesco S. Cafagna*, for the TOTEM Collaboration \\ INFN Sezione di Bari, Italy \\ E-mail: francesco.cafagna@ba.infn.it
}

The LHC energy begins to overlap with the energy range where extreme energy cosmic-ray showers are studied. Investigations of proton-proton interactions at the LHC are therefore of high importance for the study of the development of cosmic-ray showers in the atmosphere and thus for the measurements of the high-energy cosmic-ray spectra and composition.

The TOTEM (TOTal cross section, Elastic scattering and diffraction dissociation Measurement at the LHC) experiment at LHC, has been designed to measure the total proton-proton cross-section with a luminosity independent method, based on the optical theorem, and study the elastic and diffractive scattering at the LHC energy. This method relies on the capability of the simultaneous measurements of inelastic and elastic rates; in the TOTEM experiment this is possible thanks to two forward inelastic telescopes, covering the pseudorapitidy range $3.1<|\eta|<6.5$, and Roman Pot detectors, that can be inserted down to few hundred microns to the beam centre.

Thanks to dedicated runs, taken between 2011 and 2012, with special beam optics, TOTEM experiment was able to measure the elastic, inelastic and total cross-section at $\sqrt{s}=7 \mathrm{TeV}$ and $8 \mathrm{TeV}$, using the luminosity independent method, along with the pseudorapidity distribution of charged particles.

In this contribution the latest results of the TOTEM experiment will be described along with its performance and the future physics program for the LHC run 2.

The 34th International Cosmic Ray Conference,

30 July- 6 August, 2015

The Hague, The Netherlands

${ }^{*}$ Speaker. 


\section{Introduction}

The LHC energy begins to overlap with the energy range where extreme energy cosmic-ray showers are studied. Investigations of proton-proton interactions at the LHC are therefore of high importance for the study of the development of cosmic-ray showers in the atmosphere and thus for the measurements of the high-energy cosmic-ray spectra and composition. One of the most important observable: the shower maximum $\left(\mathrm{X}_{\max }\right)$, strongly depends on the inelastic proton-air crosssection and calculations of hadron-nucleus cross-sections in the Glauber-Gribov formalism require the knowledge of the inelastic pp cross-section along with the pp elastic scattering amplitude in the small $|t|$ range. In addition, all kinds of diffractive phenomena influence the development of the shower cascade and the multiplicity fluctuations of secondary hadrons [1]. Thus, the measurements of the pp cross-sections and the particle flow are of high importance for the interpretation of cosmic ray showers.

TOTEM measured the total pp cross-section at the energy of $\sqrt{s}=7 \mathrm{TeV}$ and $8 \mathrm{TeV}[2,3]$, using a luminosity, $\mathscr{L}$, independent method and validating it by comparing several methods to determine the total cross-sections $[4,5,6,7]$. The total cross-section can be obtained via the optical theorem that combines the total inelastic rate $N_{\text {inel }}$ and the total nuclear elastic rate $N_{e l}$ with its extrapolation to $t=0, \mathrm{~d} N_{e l} /\left.\mathrm{d} t\right|_{t=0}$.

$$
\sigma_{t o t}=\frac{16 \pi}{1+\rho^{2}} \frac{\left(\mathrm{d} N_{e l} / \mathrm{d} t\right)_{t=0}}{\left(N_{e l}+N_{\text {inel }}\right)}
$$

where $\rho$ is the ratio of the real to imaginary part of the hadronic scattering amplitute at $t=0$. The method requires the simultaneous measurements of the inelastic and elastic rates, as well as the extrapolation of the latter in the invisible region down to $|t|=0$. This is achieved with the experimental set-up of TOTEM which consists of two inelastic telescopes, called T1 and T2, to detect charged particles produced in inelastic pp collisions, and Roman Pot stations to detect elastically scattered protons at very small angles.

The optical theorem can be used also to derive the total and fully inclusive inelastic pp crosssection, from the measurement of the elastic scattering one:

$$
\sigma_{t o t}^{2}=\left.\frac{16 \pi}{1+\rho^{2}} \frac{\mathrm{d} \sigma_{e l}}{\mathrm{~d} t}\right|_{t=0}, \quad \sigma_{\text {inel }}=\sigma_{t o t}-\sigma_{e l}
$$

To access to the smaller $|t|$-value region, the colliding beams must have a beam divergence of not more than a few micro-radians[8]. This can be obtained by either increasing the beta function value, $\beta^{*}$, or by reducing the beam emittance, $\varepsilon$ (beam divergence $=\sqrt{\varepsilon / \beta^{*}}$ ). With a dedicated beam optics configuration $\left(\beta^{*}=90 \mathrm{~m}\right)$, TOTEM extended the measurement to $|t|$-values as low as $5 \times 10^{-3} \mathrm{GeV}^{2}$, measuring the differential elastic cross-section over a wide range of $t[7,4]$. This made the extrapolation of the differential cross-section to the optical point at $t=0$ possible, allowing, for the first time at the LHC, the determination of the elastic scattering cross-section as well as the total cross-section via the optical theorem.

Moreover, with the data collected by the very forward telescopes T1 and T2, during special runs at $\sqrt{s}=7 \mathrm{TeV}$, also the double diffractive (DD) cross-section was measured in the pseudorapidity region $4.7<\left|\eta_{\min }\right|<6.5[9]$. 

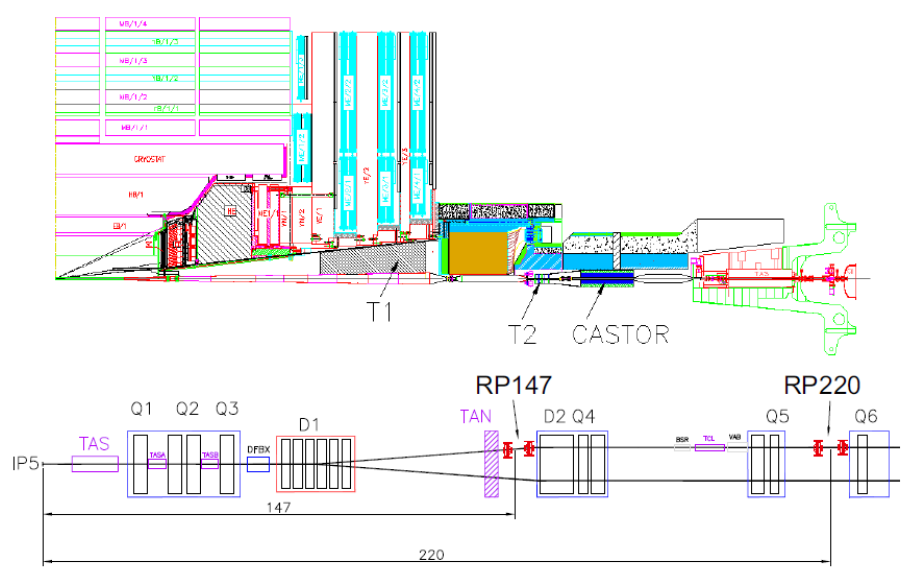

a)

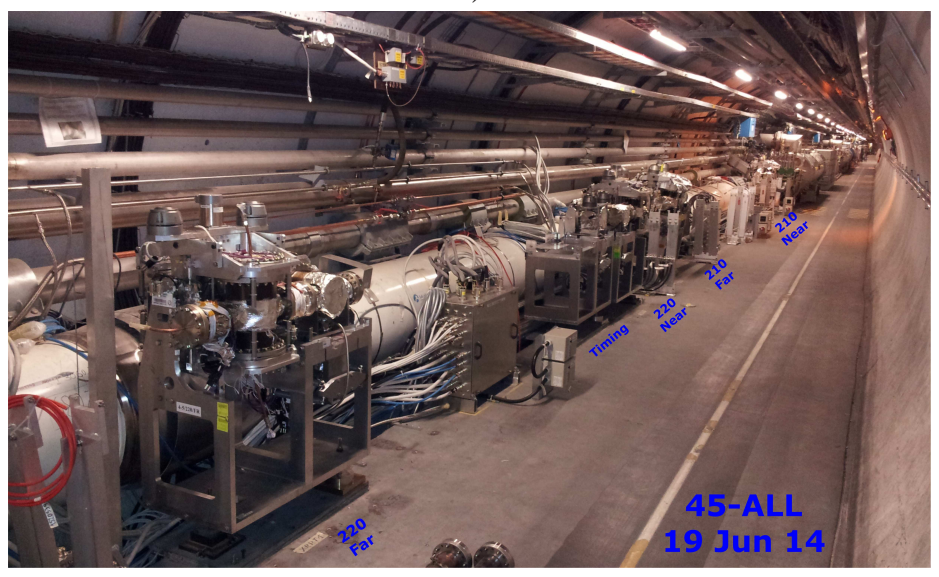

b)

Figure 1: The TOTEM experiment layout before and after the LHC Long Shutdown 1. a) The position of the inelastic telescopes: T1 and T2, in the CMS experiment (top), and the old Roman Pots locations, along the LHC beam line, at $147 \mathrm{~m}$ (RP147) and $220 \mathrm{~m}$ (RP220) (bottom). b) The new Roman Pot location along one tunnel arm. The $147 \mathrm{~m}$ stations are now installed at $210 \mathrm{~m}$, while the newly designed horizontal pots (Timing) are installed between the $220 \mathrm{~m}$ far and near.

During the special runs, TOTEM provides minimum and zero bias triggers to the CMS experiment [10], making also possibile physics studies in common between the two experiments. In particular the direct measurement of forward pseudorapidity distributions, that are also valuable in constraining theoretical models and Monte Carlo programs of the high energy air showers initiated by cosmic rays, have been measured by both experiments [11] and by TOTEM standalone [12].

\subsection{The TOTEM experiment}

The TOTEM experiment layout, before the LHC long shutdown one (LS1), is shown in Figure 1 along with the position of the inelastic telescopes and Roman Pot stations. The two inelastic telescopes consists of two arms located symmetrically on both sides of the LHC interaction point 5 (IP5): the T1 telescope with cathode strip chambers (CSC), placed at a distance of $9 \mathrm{~m}$ from IP5, covers the pseudorapidity range $3.1 \leq \eta \leq 4.7$. The T2 telescope with gas electron multiplier 


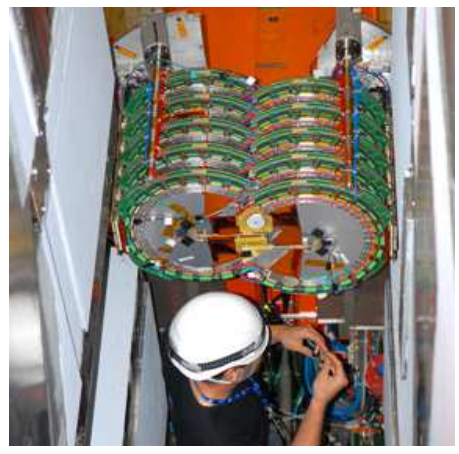

a) $\mathrm{T} 2$ detector

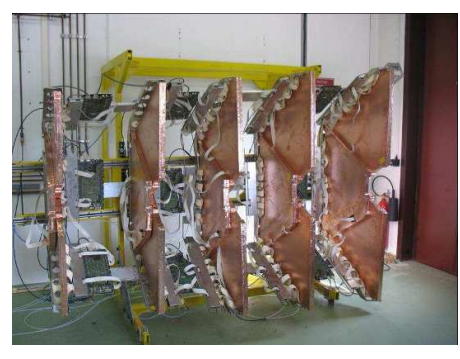

b) $\mathrm{T} 1$ detector

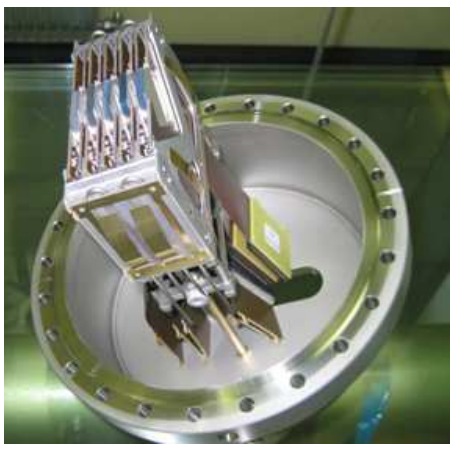

c) Roman Pot siliicon planes

Figure 2: a) One arm of the T2 GEM based inelastic telescope during the installation in IP5. b) Half of an arm of the T1 CSC based telescope before the installation. c) The silicon detector stack of a Roman Pot, as seen from the bottom cover.

(GEM) chambers, located at $\pm 13.5 \mathrm{~m}$ from IP5, covers the pseudorapidity range $5.3 \leq \eta \leq 6.5$. The pseudorapidity coverage of the two telescopes allows the detection of $95 \%$ of the inelastic events, including events with difractive mass down to $3.6 \mathrm{GeV}$. Both $\mathrm{T} 1$ and $\mathrm{T} 2$ telescopes are shown in Figure 2.

The Roman Pot (RP) stations, used for these measurements, are located symmetrically on either side of the IP5 at distances of 215-220 m from it. Each station is composed of two units (near and far, in the TOTEM jargon) separated by a distance of about $5 \mathrm{~m}$. A unit consists of 3 RPs, two approaching the outgoing beam vertically, from the top and the bottom, and one horizontally. Each $\mathrm{RP}$ is equipped with a stack of 10 silicon strip detectors, designed with the specific objective of reducing the insensitive area at the edge facing the beam to only a few tens of micrometers. This design permits to measure the proton distance from the beam center, in both coordinates perpendicular to the beam, with a precision of about $11 \mu \mathrm{m}$ [13]. The movement and the alignment of all pots are monitored with a precision better than $20 \mu \mathrm{m}$ based on track reconstruction and external alignment tools. The large lever arm between the near and the far units allows the determination of the track angle in both projections with a precision of about $5 \mu \mathrm{rad}$. The silicon detector stack of one RP is shown in Figure 2.

A more detailed description of the TOTEM detector and performance, in this configuration, can be found in $[14,15]$.

The actual layout, i.e., after the LHC LS1, is different in RP location and quantity and shown in Figure 1. In this updated configuration[16], the RP stations previously installed at $\pm 147 \mathrm{~m}$, from the interaction point, have been relocated at $\pm 210 \mathrm{~m}$, with one of the two unit, the far one, tilted around the beam axis by $8^{\circ}$. Moreover two newly designed horizontal Roman Pots, have been installed between the two station at $\pm 220 \mathrm{~m}$. This new layout has been proposed to improve the apparatus, adding timing detectors, to resolve the pileup of multiple events in the same bunch crossing, and operate the experiment at higher luminosities, adding radiation-hard silicon detectors. This program will be fulfilled by TOTEM, for the special runs with low luminosity, installing a new timing detector in a relocated vertical RP[17]. While CMS and TOTEM, join proposal called: CMS-TOTEM Precision Proton Spectrometer (CT-PPS), for the standard run with high 
luminosity[18]. The CT-PPS collaboration proposes the installation of new tracking detectors in two horizontal RP and a timing detector in one of the new horizontal. The main challenge of the CT-PPS experimental programme is the ability to operate the detectors close to the beam $(10-20 \sigma)$ at high luminosity

\subsection{Data set}

The measuremenents, reported in this contributions, are the results of the analysis of two data samples recorded in October 2011, at $\sqrt{s}=7 \mathrm{TeV}$, and July 2012, at $\sqrt{s}=8 \mathrm{TeV}$. In both cases data have been recorded during special low intensity LHC fills with $\beta^{*}=90 \mathrm{~m}$.

For the TOTEM standalone measurement of the charged particle pseudorapidity density, was used a small sub-sample of the events, collected with the T2 minimum bias trigger in July 2012, having collisions displaced by $11.25 \mathrm{~m}$ from the nominal interaction point.

In these special runs, the Roman Pots, are moved very close to the beam center, the actual distances depending on the transverse beam size value, $\sigma_{\text {beam }}$. This is possible when the beams are scraped by the LHC collimators at a distance equal to 4 times the $\sigma_{\text {beam }}$. In this configuration TOTEM took data in very clean conditions with only a few colliding bunch pairs and reached $|t|$-values down to $5 \times 10^{-3} \mathrm{GeV}^{2}$, enabling the observation of $91 \%$ of the elastic cross-section.

The RP first-level trigger is based on track segment, in the near or the far unit. A coincidence between a proton on the left and the right side of the interaction point is requested in the elastic double-arm signature, in the vertical RP detectors, in either of the two diagonals (left top - right bottom or left bottom - right top). The RP detector was designed for the characteristics of a beam optics with large $\beta^{*}$. With this optics configuration most of the elastically scattered protons can be measured by the vertical RP. Elastically scattered protons cross the RP detectors close to the vertical plane, while diffrative protons are deviated to positive $x$-values in the vertical detectors. Defining in this way a nice topological criterion for selecting elastic candidate events [8].

Moreover a minimum bias trigger, requiring at least one track candidate in one of the T2 detector arm, was used during these runs, making possible the direct measurement of the inelastic [5] and DD cross-section [9], along with the determination of the charged particle pseudorapidity density $[11,12]$. This trigger is available to the CMS experiment for the common data taking, along with a zero bias trigger, obtained by triggering on random bunch crossings, that has been used to evaluate the trigger efficiency and to cross-check the pileup probability estimate.

\section{Results}

Given the various methods of measuring elastic and inelastic rates, it was possible to perform completely different estimation of the inelastic, elastic and total cross-sections, comparing the results for a better understanding of the systematic uncertainties and double checks.

By extrapolating the differential elastic cross-section to the optical point $|t|=0$ and using the optical theorem, the total and inelastic cross-sections were also derived [4, 2]. While from the direct measurement of the inelastic rates the inelastic cross-section was determined and compared with the one deduced from the difference between the total and the elastic cross section [5].

The cross-sections obtained using the luminosity independet method of eq. 1.1, during the special run with $\beta^{*}=90 \mathrm{~m}$, are reported in Table 1 and shown in Figure 3, along with the ones obtained 
Table 1: Summary of the cross-sections measured using a luminosity, $\mathscr{L}$, independent method and the double diffractive (DD) one, as measured in the forward region. The full systematic uncertainty is reported for each measurements, see [7, 2, 3, 9] for a detailed description of this uncertainties.

\begin{tabular}{|l|c|c|}
\hline & $\begin{array}{c}\mathscr{L} \text { independent at } \\
\sqrt{s}=7 \mathrm{TeV} \text {, eq. } 1.1\end{array}$ & $\begin{array}{c}\mathscr{L} \text { independent at } \\
\sqrt{s}=8 \mathrm{TeV} \text {, eq. } 1.1\end{array}$ \\
\hline$\sigma_{\text {tot }}(\mathrm{mb})$ & $98.0 \pm 2.5$ & $101.7 \pm 2.9$ \\
\hline$\sigma_{\text {inel }}(\mathrm{mb})$ & $72.9 \pm 1.5$ & $74.7 \pm 1.7$ \\
\hline$\sigma_{\text {el }}(\mathrm{mb})$ & $25.1 \pm 1.1$ & $27.1 \pm 1.4$ \\
\hline \hline & \multicolumn{2}{|c|}{$\sqrt{s}=7 \mathrm{TeV}, 4.7<\left|\eta_{\min }\right|<6.5$} \\
\hline$\sigma_{D D}(\mu \mathrm{b})$ & \multicolumn{2}{|c|}{$116 \pm 25$} \\
\hline
\end{tabular}

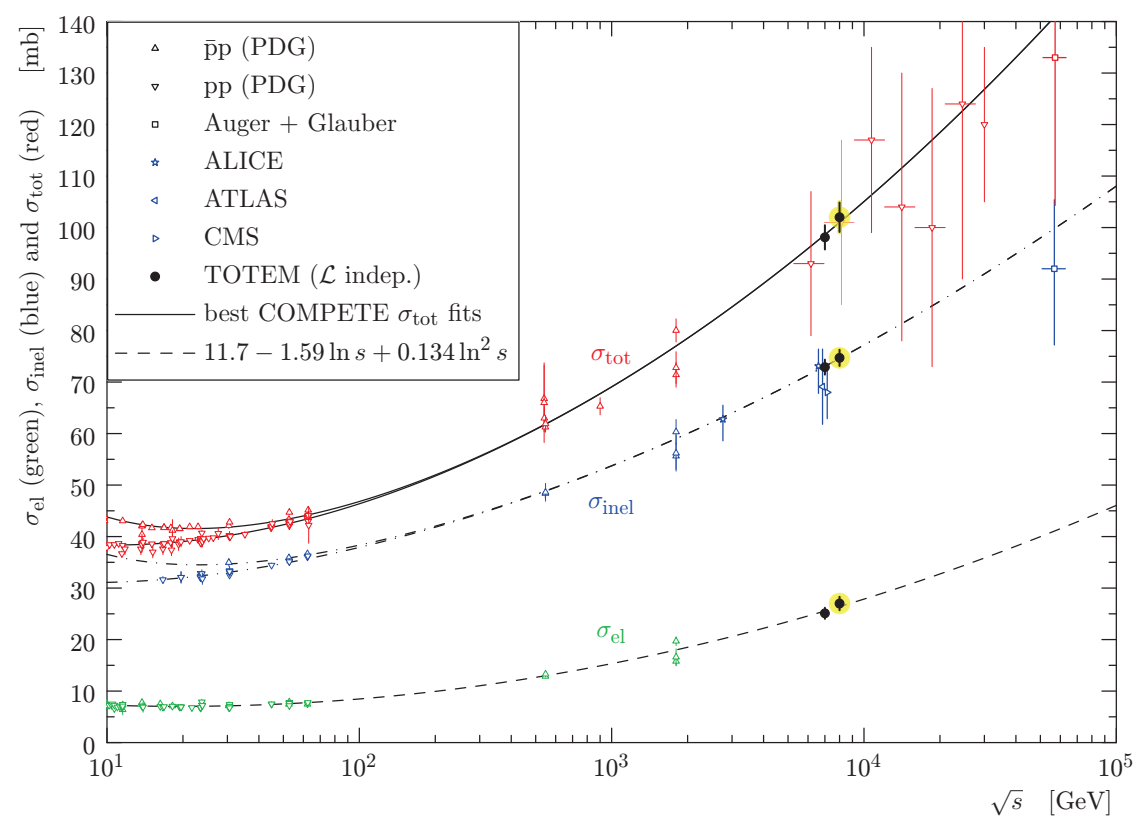

Figure 3: A compilation of $[2,20,22,21,19]$ the total $\left(\sigma_{t o t}\right)$, inelastic $\left(\sigma_{\text {inel }}\right)$ and elastic $\left(\sigma_{e l}\right)$ cross-sections measurements: the TOTEM measurements using the luminosity independent method are the black points. The continuous black lines (lower for pp, upper for $\bar{p}$ ) represent the best fits of the total cross-section data by the COMPETE collaboration [24]. TOTEM results at $\sqrt{s}=8 \mathrm{TeV}$ are highlighted. The dashed line results from a fit of the elastic scattering data. The dash-dotted lines refer to the inelastic cross-section and are obtained as the difference between the continuous and dashed fits.

by other experiments at LHC [20, 22, 21] and by the AUGER Collaboration [23]. The good agreement between TOTEM measurements and the COMPETE fit, extrapolated from lower energies [24], can be noted. In the table the total systematic uncertainty has been reported, obtained combining in quadrature all the systematic uncertainties, taking into account the correlations between contributions. For a more detailed description and discussion of the systematic uncertainties for these measurements see $[7,2,3]$ and references therein. For the $\rho$ parameter value the COMPETE [24] preferred-model extrapolation of $0.141 \pm 0.007$ was chosen. 


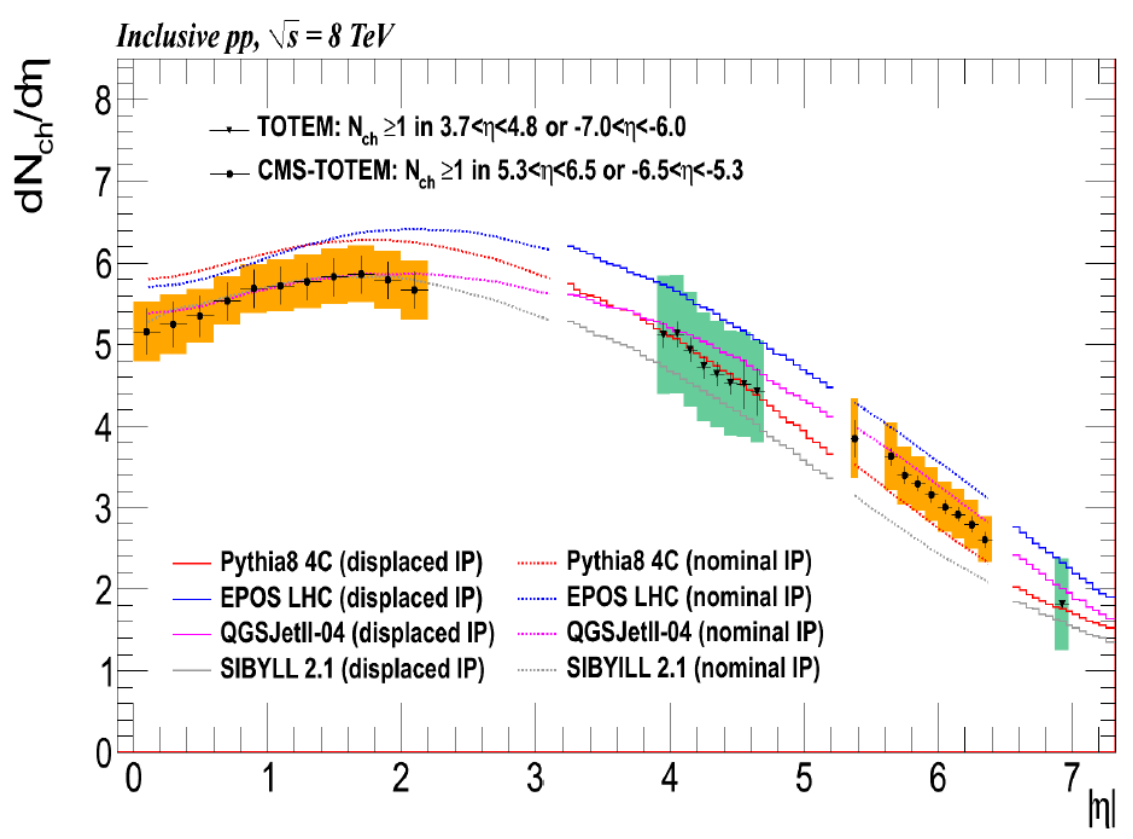

Figure 4: Charged particle pseudorapidity distributions obtained in pp collisions at $\sqrt{s}=8 \mathrm{TeV}$ for inelastic events. The coloured bands show the combined systematic and statistical uncertainties and the error bars represent the $\eta$-uncorrelated uncertainties. Points in the ranges: $3.9<|\eta|<4.7$ and $6.95<|\eta|<6.9$, have been measured by $\mathrm{T} 2$ with the displaced interaction point (IP) [12], while the others by CMS and TOTEM with the nominal IP [11]. Measurements are compared in each $\eta$ region to the corresponding prediction from PYTHIA8 (tune 4C), SIBYLL 2.1, EPOS (tune LHC), and QGSJETII-04.

In the same Table 1, also the DD cross section is reported. This cross/section has been measured for the first time in this $\eta$ range. The TOTEM measurement is $\sigma_{D D}=131 \pm 22 \mu \mathrm{b}$, for events having at least one particle in both sides of $5.3<|\eta|<6.5$ and no particle within $3.1<|\eta|<4.7$. The result was deduced with PYTHIA for events that have both diffractive systems with $4.7<|\eta|<6.5$.

\section{Conclusions}

TOTEM has measured, for the first time at the LHC, the total proton-proton cross section at both $\sqrt{s}=7 \mathrm{TeV}$ and $\sqrt{s}=8 \mathrm{TeV}$, using a luminosity independent method. The method was validated comparing the elastic and inelastic cross sections measured in independent ways. Furthermore, double diffractive cross section has been measured in an $\eta$ range where it has never been determined before. Furthermore quantity that are relevant in cosmic rays studies, like the charge particle pseudorapidity distributions, has been measured in the forward region.

After the LS1, TOTEM will join forces with CMS, creating a combined apparatus with the largest $\eta$ coverage and with the most performing two-arm proton spectrometer ever built at a collider.

Acknowledgment:We are grateful to the beam optics development team for the design and the successful commissioning of the high $\beta^{*}$ optics and to the LHC machine coordinators for scheduling the dedicated fills. 
This work was supported by the institutions listed on the front page and partially also by NSF (US), the Magnus Ehrnrooth foundation (Finland), the Waldemar von Frenckell foundation (Finland), the Academy of Finland, the Finnish Academy of Science and Letters (The Vilho, Yrjö and Kalle Väisälä Fund),the OTKA grant NK 73143 (Hungary) and by the NKTH-OTKA grant 74458 (Hungary).

\section{References}

[1] D. D‘Enterria et al., Astropart. Phys., 35 (2011) 98.

[2] G. Antchev et al. (TOTEM Collaboration), EPL, 101 (2013) 21004, doi:10.1209/0295-5075/101/21004.

[3] G. Antchev et al., Phys. Rev. Lett. 111 (2013) 012001, doi: 10.1103/PhysRevLett.111.012001.

[4] G. Antchev et al. (TOTEM Collaboration), EPL, 101 (2013) 21002, doi:10.1209/0295-5075/101/21002.

[5] G. Antchev et al. (TOTEM Collaboration), EPL, 101 (2013) 21003, doi:10.1209/0295-5075/101/21003.

[6] G. Antchev et al. (TOTEM Collaboration), EPL, 96 (2011) 21002, doi: 10.1209/0295-5075/96/21002.

[7] G. Antchev et al. (TOTEM Collaboration), EPL, 95 (2011) 41001, doi: 10.1209/0295-5075/95/41001.

[8] G. Anchev et al. (TOTEM Collaboration), New J. Phys. 16 (2014) 103041, doi:10.1088/1367-2630/16/10/103041.

[9] G. Antchev et al., Phys. Rev. Lett. , 111 (2013) 262001, doi: 10.1103/PhysRevLett.111.262001.

[10] CMS Collaboration, JINST 3, S08004 (2008). doi:10.1088/1748-0221/3/08/S08004.

[11] The CMS and TOTEM Collaborations, EPJC 74 (2014) 3053, doi:10.1140/epjc/s10052-014-3053-6.

[12] G. Antchev et al. (TOTEM COllaboration), EPJC 75 (2015) 126, doi:10.1140/epjc/s10052-015-3343-7.

[13] E. Alagoz et al., JINST, 8, P06009 (2013), doi:10.1088/1748-0221/8/06/P06009.

[14] G. Anelli et al. (TOTEM Collaboration), JINST, 3, S08007 (2008), doi:10.1088/1748-0221/3/08/S08007.

[15] G. Antchev et al., Int. J. Mod. Phys. A 28, 1330046 (2013), doi: 10.1142/S0217751X13300469.

[16] TOTEM Collaboration, TOTEM Upgrade Proposal, CERN-LHCC-2013-009, (2013), url: https://cds.cern.ch/record/1554299.

[17] TOTEM Collaboration, Timing Measurements in the Vertical Roman Pots of the TOTEM Experiment, CERN-LHCC-2014-020, (2014), url: https://cds.cern.ch/record/1753189 .

[18] M. Albrow et al. (CMS-TOTEM Collaboration), CMS-TOTEM Precision Proton Spectrometer, CERN-LHCC-2014-021, (2014), url: https://cds.cern.ch/record/1753795 .

[19] K. Nakamura et al., (Particle Data Group), J. Phys. G37, 075021 (2010).

[20] ALICE Collaboration, EPJC 73, 2456 (2013), doi:10.1140/epjc/s10052-013-2456-0.

[21] ATLAS Collaboration, Nature Commun. 2 (2011) 463.

[22] CMS Collaboration, Phys. Lett. B, 722, 1-3 (2013), doi:10.1016/j.physletb.2013.03.024.

[23] P. Abreu et al., Phys. Rev. Lett. , 109 (2012) 062002, doi:10.1103/PhysRevLett.109.062002.

[24] J. R. Cudell et al. (COMPETE Collaboration), Phys. Rev. Lett. 89, 201801 (2002). 